\title{
La connaissance des grotte medicee
}

Nouvelles approches à partir des archives de l'époque des HabsbourgLorraine

The knowledge of the Medici grottoes: new approaches from the archives of the Habsburg-Lorraine period

\section{Giulia Cicali}

\section{(2) OpenEdition}

\section{Journals}

Édition électronique

URL : http://journals.openedition.org/artefact/5333

DOI : $10.4000 /$ artefact.5333

ISSN : 2606-9245

Éditeur :

Association Artefact. Techniques histoire et sciences humaines, Presses universitaires du Midi

Édition imprimée

Date de publication : 15 juillet 2020

Pagination : 53-71

ISBN : 978-2-8107-0691-4

ISSN : 2273-0753

\section{Référence électronique}

Giulia Cicali, «La connaissance des grotte medicee », Artefact [En ligne], 12 | 2020, mis en ligne le 21 décembre 2020, consulté le 23 décembre 2020. URL : http://journals.openedition.org/artefact/5333 ; DOI : https://doi.org/10.4000/artefact.5333

\section{(c) (†) $\odot$}

Artefact, Techniques, histoire et sciences humaines est mise à disposition selon les termes de la Licence Creative Commons Attribution - Pas d'Utilisation Commerciale - Pas de Modification 4.0 International. 


\title{
La connaissance des grotte medicee
}

\author{
Nouvelles approches à partir des archives de l'époque \\ des Habsbourg-Lorraine
}

Giulia Cicali

\section{Résumé}

Les archives produites par l'administration des Habsbourg-Lorraine en Toscane, après que cette Maison avait hérité en 1737 de l'ancien grand-duché des Médicis, ont longtemps été considérées comme des sources tardives et indirectes. Pourtant, elles révèlent la manière dont le patrimoine des Médicis a été géré par la nouvelle dynastie. En ce qui concerne la conservation (et la destruction) des sculptures, des fontaines et des grottes de jardins qui entouraient les villas médicéennes, ce sont tout particulièrement les archives conservées dans les fonds du Scrittoio Fortezze e Fabbriche et du Scrittoio Regie possessioni des Archives d'État de Florence qui méritent notre attention. Elles contiennent des actes relatifs à l'entretien et à la restauration des anciens domaines des Médicis aux XVIII et $\mathrm{XIX}^{\mathrm{e}}$ siècles. Nous proposons ici une analyse de ce patrimoine dans son ensemble, en portant une attention particulière aux témoignages concernant les prestigieux jardins et notamment des grotte medicee.

\section{Mots-clés}

Habsbourg-Lorraine, Médicis, villa, Castello, Pratolino, Boboli

95 Giulia Cicali, « La connaissance des grotte medicee. Nouvelles approches à partir des archives de l'époque des Habsbourg-Lorraine », Artefact, 12, 2020, p. 53-71. 


\section{The knowledge of the Medici grottoes: new approaches from the archives of the Habsburg-Lorraine period}

\section{Abstract}

The archives produced by the Habsburg-Lorraine administration in Tuscany, after inheriting the former Grand Duchy of Medici in 1737, have long been considered late and indirect sources. However, they do reveal the way in which the Medici heritage was managed by the new dynasty. With regard to the conservation (and destruction) of the sculptures, fountains and garden grottoes that surrounded the Medici villas, it is particularly the archival records kept in the Scrittoio fortezze e fabbriche and the Scrittoio regie possessioni of the State Archives of Florence that deserve our attention. They contain acts relating to the maintenance and restoration of the ancient Medici estates in the $18^{\text {th }}$ and $19^{\text {th }}$ centuries. Here we offer an analysis of this heritage as a whole, with particular attention to the testimonies concerning the prestigious gardens and in particular the Medici grottoes.

\section{Keywords}

Habsbourg-Lorraine, Medici, villa, Castello, Pratolino, Boboli 


\section{Introduction}

Suite à la prise du pouvoir de Cosme Ir de Médicis (1537) ${ }^{1}$, à Florence se développe une véritable cour princière, sous l'influence de la culture napolitaine de la duchesse Éléonore de Tolède. Bien qu'elle n'ait jamais été institutionnalisée auparavant, elle devient progressivement un élément incontournable de l'exercice du pouvoir ${ }^{2}$. Parallèlement, à partir de 1550, le duc installe sa demeure officielle dans la reggia du Palais Pitti, face au jardin de Boboli, et il se déplace souvent dans d'autres villas des alentours de Florence ${ }^{3}$. Ces espaces, avec leurs grottes ${ }^{4}$, se transforment en scène de représentation " théâtrale " destinée à l'exaltation des souverains devant les courtisans locaux et les étrangers en visite. Les uns comme les autres entrent dans un univers parallèle, dans lequel non seulement ils assistent mais ils participent eux-mêmes aux jeux des Médicis - entraînés par les automates qui animent ces lieux auxquels ils sont (sous leurs propres yeux) assimilés. L'art médicéen, face au défi de la mimesis de la nature ${ }^{5}$, y avait recréé une vie artificielle jouant sur la dualité thématique du mythe et de la réalité. Et comme le peuple avait été séduit par le prince, plusieurs œuvres glorifiaient ses triomphes, telles que les célèbres représentations d'Orphée qui charmait les animaux.

Tout ce patrimoine inclut, notamment, les jardins palatiaux avec leur richissime collection de statues dont héritèrent les Habsbourg-Lorraine en 1737, à la mort de Jean-Gaston de Médicis. Lorsque la dynastie souveraine change, que s'ouvre une époque nouvelle, que l'opulence des Médicis est remplacée par les idées portées par les Habsbourg-Lorraine, le statut de cette cour d'automates est questionné. Ces changements provoquent

1. Cet article est issu de la communication intitulée «La connaissance des grotte medicee : notes sur les problématiques et les possibilités de recherche ", que j’ai eu le plaisir de présenter le 2 novembre 2018 à la mairie de Noisy-le-Roi, lors du $2^{\mathrm{e}}$ séminaire La Renaissance des grottes.

2. Côme Ir de Médicis (1519-1574) devient duc de Florence en 1537, puis il obtient le nouveau titre de grand-duc en 1569 grâce à une bulle du pape Pie V ; c'est avec lui et ses premiers successeurs, Francois $I^{\text {er }}$ et Ferdinand I Ir, que la cour se structure. Fasano Guarini, 1984 ; Chauvineau, 2004 .

3. Pour témoigner de l'importance de ce patrimoine, exemple de l'influence exercée par la famille Médicis sur la culture européenne, douze villas Médicis ont été inscrites sur la liste du patrimoine mondial de l'UNESCO en 2013 : http://whc.unesco.org/en/list/175; Zangheri, 2015.

4. Pour un répertoire de grottes dans la région toscane : Cazzato, Fagiolo, Giusti, 2001.

5. En particulier, sur la représentation de la natura naturans et le rapport aux sciences naturelles : Morel, 1998. 
un problème complexe de transmission, d'interprétation et de conservation d'un ensemble de biens profondément ancrés dans l'histoire toscane. Les archives qui conservent la mémoire de ces richesses reflètent ce nouveau rapport aux ouvrages du passé. Pour approfondir cette question, il est cependant nécessaire d'aller au-delà des principales sources sur lesquelles se fonde traditionnellement la connaissance de ces grottes, c'est-à-dire les actes produits à l'époque de leur création au temps des Médicis comme les livres des comptes, qui témoignent des travaux effectués, les descriptions littéraires dédiées à ces villas, les journaux de voyage des visiteurs qui les avaient observées, ou bien encore les réflexions théoriques ${ }^{6}$, auxquelles il faut ajouter la documentation graphique, des vues de Giusto Utens (1599) aux dessins de Giovanni Guerra ${ }^{7}$. En l'espèce, c'est plutôt l'ensemble des documents produits par l'administration des Habsbourg-Lorraine qui apporte un éclairage original en montrant la façon dont les grottes réalisées par les Médicis ont été gérées par la suite. Tout particulièrement, la documentation graphique conservée dans les fonds du Scrittoio delle Fortezze e Fabbriche et du Scrittoio delle Regie possessioni des Archives d'État de Florence constitue un ensemble de sources apparemment secondaires ${ }^{8}$ : elle ouvre néanmoins la porte à une microhistoire de fonds d'archives qui méritent d'être relus.

\section{Les archives du Scrittoio delle Fortezze e Fabbriche}

Dans le fonds du Scrittoio delle Fortezze e Fabbriche [le bureau des forteresses et des bâtiments] $]^{9}$, la section des Fabbriche lorenesi contient des dossiers relatifs à l'entretien et à la restauration des forteresses et des bâtiments

6. À titre d'exemple, pour la villa de Pratolino, voir les plus importantes descriptions littéraires : Gualterotti, 1579 ; De’ Vieri, 1586 ; Montaigne, éd. 1992 ; au sujet des réflexions théoriques : le chapitre Del giardino di un re du traité l'Agricoltura sperimentale de Agostino Del Riccio (Heikamp, 1981, p. 59-123), et l'ouvrage de Salomon de Caus, 1624. Pour une bibliographie ample et structurée : Brunon, 2001 ; Valdaré, 2003.

7. Becattini, 2005.

8. Je remercie Francesco Martelli de m'avoir signalé l'importance de ces fonds. Bien évidemment, certains documents ont déjà été publiés et étudiés et il n’est pas possible ici de répertorier toutes les références relatives aux actes que nous discuterons. Nous serons plus intéressés par une réflexion dédiée à l'ensemble des sources graphiques, ses caractéristiques et son importance, sa relation avec la documentation écrite qui, à l’origine, lui était associée.

9. Martelli, 2006. 
des Médicis entre le XVIII ${ }^{\mathrm{e}}$ et le XIX ${ }^{\mathrm{e}}$ siècle. Il s'agit d'une source exceptionnelle car ce fonds conserve les textes d'archives qui concernent leurs villas et leurs jardins, complétés par une importante documentation graphique ${ }^{10}$.

Pour la villa de Poggio Imperiale et son jardin ${ }^{11}$, un fascicule témoigne, par exemple, de l'état des fontaines du jardin en $1820^{12}$. Dans un autre dossier daté de $1779^{13}$ qui concerne le projet d'une tromba d'acqua, se trouve le dessin d'une pompe hydraulique servant à puiser l'eau d'un pozzo (puits) situé près de la grotte, dont nous connaissons, ainsi, indirectement, l'état (Fig. 1). D'ailleurs, dans ce cas, nous sommes confrontés à une difficulté majeure que nous allons retrouver souvent : puisque le dessin ne donne aucune explication concernant la destination de cette invention, il n'aurait pas été possible de retrouver la fonction de ce mécanisme s'il n'avait pas été relié à une documentation écrite.

Remontant au mois de juillet 1791, lors de la préparation d'une fête pour la cour et de la villeggiatura d'automne du grand-duc ${ }^{14}$, un autre dossier, relatif à Poggio a Caiano, renvoie à l'aspect ludique des ouvrages aménagés dans les jardins, ce qui concerne aussi, bien entendu, les grottes, où la présence de scherzi d'acqua (jeux d'eau) visait à créer un émerveillement et un élan émotionnel qui mettaient le spectateur en relation étroite avec les œuvres d'art. Ce texte signale qu'on doit transporter dans le parc de la villa des Macchine giocose (des machines ludiques) provenant des Cascine : il s'agit d'une giostra degli asini (un carrousel avec des ânes), d'une giostra dei cavalli e sedie (un carrousel de chevaux et de chaises) et d'un arcolaio volante (un rouet volant). Ces machines sont représentées dans les plans qui accompagnent ces documents et qui indiquent leur emplacement, mais, là aussi, l'image seule ne suffirait pas pour comprendre l'ensemble.

10. L'analyse de cette documentation est désormais assez accessible grâce au fait qu'en 1987 Leonardo Rombai, Diana Toccafondi et Carlo Vivoli ont publié une étude dédiée aux fonds cartographiques des archives de l'État de Florence (Rombai, Toccafondiet et Vivoli, 1987), ce qui a permis de redécouvrir ce patrimoine. Ensuite, les fonctionnaires des archives ont aussi procédé à une numérisation qui a rendu plus aisée la vision de la documentation conservée dans les cartons. En revanche, il est encore difficile de consulter les piante sciolte regie possiessioni, c'est-à-dire la documentation en grand format des possessions royales.

11. Sur la collezione fiorentina di antichità qui ornaient la villa, fondamentale pour l'étude des collections des Médicis et leur destination d'origine : Capecchi, Lepore, Saladino, 1979.

12. Archives d'État de Florence (dorénavant ASFi), Fabbriche Lorenesi, 2072-20 (1820).

13. ASFi, Fabbriche Lorenesi, 1991-48 (1779).

14. ASFi, Fabbriche Lorenesi, 2010-114 (1791). Bardazzi-Castellani, 1982, vol. II, p. 682-683. 


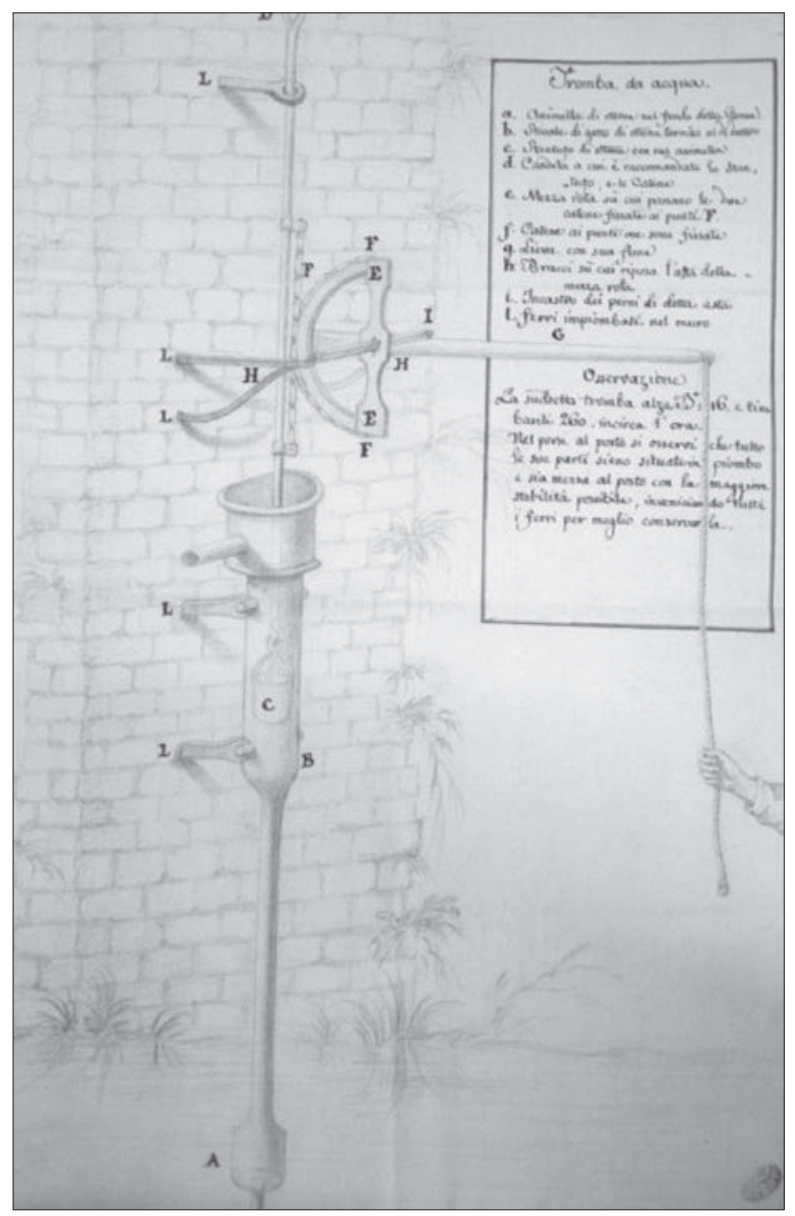

Fig. 1. - Poggio Imperiale : dessin d'une pompe hydraulique

Florence, Archives d'État, fabbriche Lorenesi, 199148 (1779).

\section{Cliché Giulia Cicali}

Le dessin illustre à la fois un élément éphémère (le carrousel) et un élément permanent, et c'est grâce aux seules notes écrites que nous savons comment ces mécanismes étaient conservés et protégés dans les magasins dont on parle, et où ils étaient installés à l'occasion des fêtes, c'est-à-dire sur la pelouse devant la villa, par l'architecte Giovan Battista Ruggieri. En même temps, Lugi Bartolini, du secrétariat de la Couronne, commande à Ruggieri de construire également des estrades en bois pour le Parter du 
Théâtre de la Villa, afin qu'il puisse accueillir jusqu'à 400 spectateurs. Cet exemple témoigne d'une part d'une pratique de réutilisation des œuvres et des matériaux dans les chantiers autrefois gérés par les Médicis et désormais sous la responsabilité des Habsbourg-Lorraine, d'autre part de la disposition des œuvres dans le parc ${ }^{15}$.

Deux dossiers, datés de 1816 et de $1822^{16}$, concernent les livraisons de plantes destinées à la villa de Castello, qui peuvent sembler des actes d'importance secondaire, mais qui contiennent aussi un schéma du jardin où l'on mentionne la grotte ${ }^{17}$. Les dessins et les textes relatifs à la disposition de ces plantes contiennent des informations sur son état à cette époque. De même, pour ce qui concerne Boboli, c'est un dossier de $1820^{18}$, relatif à la connexion du réseau hydraulique royal à une habitation privée voisine de la grotte, qui indique comment elle était conservée à cette date.

Parmi les actes qui témoignent des travaux réalisés dans le parc de Pratolino à la fin du XVIII ${ }^{\mathrm{e}}$ siècle, un dossier concernant la grotte de la Samaritaine est particulièrement intéressant ${ }^{19}$. Il contient les rapports et les dessins techniques que l'ingénieur Giuseppe Del Rosso a remis, en mars 1794, au soprintendente, Onofrio Boni, lorsque, sous le gouvernement des Habsbourg-Lorraine, on a démantelé ce qui restait de cette résidence des Médicis (Fig. VI et VII, cahier couleur). En effet, dès 1764, avec l'arrivée du grand-duc Pierre-Léopold, les nouveaux gouvernants s'intéressent surtout aux ouvrages d'utilité publique et récusent toute dépense inutile : ainsi commencent la dépossession du mobilier le plus précieux

15. En outre, sur les travaux du XviII ${ }^{\mathrm{e}}$ et du XIX ${ }^{\mathrm{e}}$ siècle de la Villa de Poggio a Caiano : Meddri, Mazzoni, de Vico Fallani, 1986, p. 15 ; Galetto, 2018, p. 258-260.

16. ASFi, Fabbriche Lorenesi, 2058-3 (1816) ; 2081-32 (1822).

17. La villa de Castello et son domaine étaient si célèbres que Michel de Montaigne est aussi allé les voir après avoir visité Pratolino. Ici, il a été frappé par la Grotte des animaux, qu'il a ainsi écrit : " Il y a aussi là une belle grotte, où il se voit toute sorte d'animaux représentés au naturel, rendant qui par le bec, qui par l'aise, qui par l'onge ou l'oreille ou le naseau, l'eau de ces fontaines " (Michel de Montaigne, éd. 1992, p. 84). Récemment restaurée, cette grotte fait l'objet d'une importante bibliographie. Au sujet des transformations du parc entre le XVII ${ }^{\mathrm{e}}$ et le XVIII ${ }^{\mathrm{e}}$ siècle voir, notamment, Acidini-Galletti, 1992, p. 77-87. Parmi les études les plus récentes : Capecchi, 2017 et les articles contenus dans le numéro thématique de Opus Incertum (Ferretti, 2018), en particulier Marta Castellini, dans l'article consacré à la limonaia, a reconstruit les transformations qui ont eu lieu au fil du temps dans ce secteur du jardin, en analysant les plans historiques (Castellini, 2018). 18. ASFi, Fabbriche Lorenesi, 2077- $91 / 4$ (1821).

19. ASFi, Fabbriche Lorenesi, 2015-59 (1794) ; Dezzi Bardeschi, 1986 ; Zangheri, 1987, vol. I, p. 60-71 et 280-282, vol. II, p. 38, fig. 61 (Atlante). 
de Pratolino (vers 1779) et la démolition d'une partie des bâtiments. Entre 1786 et 1789, on n'emploie plus les eaux pour les fontaines et les grottes, mais pour une fabrique de toiles et cordes. En 1798, Giuseppe Manetti évalue l'état d'abandon de la villa pour Ferdinand III de Lorraine et enfin, dans les années 1820, l'ingénieur Joseph Frietsch détruit la villa des Médicis et plusieurs pavillons du parc pour y construire d'autres bâtiments, ce qui donne naissance à un nouveau parc romantique à l'anglaise ${ }^{20}$. Ce dossier est donc l'un de derniers témoignages de ces grottes avant leur destruction. Comme l'avaient déjà signalé Marco Dezzi Bardeschi et Luigi Zangheri, c'est dans ce contexte que l'ingénieur Giuseppe Del Rosso écrit une lettre, le 8 mars 1794, dans laquelle il révèle l'intention de faire venir à Florence deux colonnes de verde antico (vert ancien) pour soutenir la voûte de la Grotte de Pratolino, tout comme une baignoire, utilisée comme salle de bains, in granito rosso egiziano (en granit rouge égyptien) existant dans la grotte souterraine voisine de la Samaritaine. Dans sa réponse, Onofrio Boni déclare que l'on doit :

remplacer non seulement un support de la voûte mais quelque chose d'analogue à la décoration déjà existante, car quel que soit le sort de cette villa royale, ce serait un mal de ne pas faire - autant que nous pouvons - quelque chose qui soit digne de la célébrité de cette grotte qui sera la dernière à périr [...] louée il y a déjà deux siècles par le célèbre Michel de Montaigne et avec raison puisque Pratolino était peut-être le modèle de ce qui a été postérieurement reproduit ailleurs en plus grand ${ }^{21}$.

L'exemple qu'il cite en témoignage de la gloire de Pratolino est tout à fait correct : Michel de Montaigne visita la villa de lors de son voyage en Italie (1580-1581). À une époque où le chantier n'était pas encore achevé, il saisit déjà l'importance des défis techniques et artistiques surmontés par le fils et premier successeur de Come I Ir, le grand-duc François I ${ }^{\text {er22 }}$, grâce

20. Berti, 1967, p. 91 ; Dezzi Bardeschi, 1986 ; Zangheri, 1986 ; Conforti, 1980.

21. "sostituire non solo un sostegno alla volta ma qualche cosa di analogo alla decorazione già esistente poiché qualunque possa essere in appresso il destino di quella regia villa sarebbe un danno il non far-per quanto si può- cosa degna della celebrità di quella grotta che sarà l'ultima a perire $[\ldots]$ encomiata già due secoli fa dal celebre Michele de Montaigne e con ragione giacché Pratolino forse è stato il modello di quanto si è fatto posteriormente in questo genere altrove più in grande ", ASFi, Fabbriche Lorenesi, 2015-59 (1794).

22. Berti, 1967 ; Benzoni, 1997. 
à son ingénieur Buontalenti ${ }^{23}$, «car il est le Prince soigneux un peu de l'alchimie et des arts mécaniques, et surtout un grand $\operatorname{architecte}^{24} »$. Ce qui l'a le plus surpris, ce sont les inventions hydrauliques et les jeux d'eau. Il déclare notamment que :

il y a de miraculeux une grotte à plusieurs demeures et pièces : cette partie surpasse tout ce que nous ayons jamais veu ailleurs. Elle est encroustée et formée partout de certaine matière qu'ils disent estre apportée de quelque montaignes ${ }^{25}$; et l'on cousue à tout des clous imperceptiblement. Il y a non seulement de la musique et harmonie qui se fait par le mouvement de l'eau, mais encore le mouvement de plusieurs statues et portes à divers actes que l'eau esbranle, plusieurs animaux qui s'y plongent pour boire, et choses semblables. À un seul mouvement toute la grotte est pleine d'eau, tous les sieges vous rejallissent l'eau aux fesses; et fuyant de la grotte, montant contremont les escaliers du chasteau, il sort de deux en deux degrés de cet escalier, qui veut se donner ce plaisir, mille filets d'eau qui vous vont baignant jusques au haut du logis. La beauté et la richesse de ce lieu ne se peut representer par le $\mathrm{menu}^{26}$.

Onofrio Boni (1739-1818), était conscient à la fois de la valeur de cet héritage en cours de disparition, et du caractère inéluctable de celle-ci. Cet homme est presque méconnu aujourd'hui. Or il requiert toute notre attention $^{27}$. En effet, deux siècles plus tard, la fortune des grottes de Pratolino en Europe, dont il avait pris la mesure, a été reconnue par les chercheurs $^{28}$. Antiquaire, architecte ${ }^{29}$, surintendant des fortezze e fabbriche

23. Dans son journal, Michel de Montaigne décrit la villa de Pratolino comme « un palais que le Duc de Florence y a basti depuis douze ans, où il employe tous ses cinq sens de nature pour l'embelir. Il semble qu'expres il ait choisi une assiette incommode, sterile et montueuse, voire et sans fontaines, pour avoir cet honneur de les aller querir à cinq milles de là, et son sable et chaux à autres cinq milles " (Montaigne, 1992, p. 79).

24. Montaigne, 1992 , p. 81

25. La pierre ponce.

26. Montaigne, 1992 , p. 79

27. Bonfioli, 1971 ; Di Croce, 2002 ; Di Croce, 2005.

28. Zangheri, 1985 ; Zangheri, 1986 ; Brunon et Mosser, 2017.

29. Élu Accademico del disegno à Florence en 1778, puis membre de l'Accademia del disegno de Rome (Académie de Saint-Luc) en 1796. En 1793, il est directeur du Scrittoio delle regie possessioni, chargé de la Surintendance générale de tous les palais, des jardins royaux et des bâtiments de toute la Toscane destinés au service militaire et civil, avec le titre de Directeur de la Fabbriche del Granduca 
de 1793 à 1818 (hormis pendant la période de l'occupation française), Onofrio Boni était aussi critique littéraire, journaliste et membre de la cour grand-ducale. Il avait été envoyé par le grand-duc Pierre-Léopold à Rome pour compléter sa formation, où il s'était lié aux artistes et aux érudits locaux. Ami de l'abbé Luigi Lanzi ${ }^{30}$, il avait écrit son éloge. Il n'était pas un simple administrateur, mais également un technicien de haut niveau dans la tradition séculaire des soprintendenti alle regie fabbriche depuis l'origine de ce bureau.

\section{Les archives des regie possessioni}

D'autres questions se posent en ce qui concerne le fonds des Scrittoio delle regie possessioni [le bureau des domaines royaux] qui contient une autre collection importante de plans et de dessins. Ici, la documentation graphique a été dissociée des archives manuscrites car, au cours du XIX ${ }^{\mathrm{e}}$ siècle (et parfois auparavant), pour mieux conserver les projets et les plans, ils ont été séparés de la documentation écrite qui autrefois les accompagnait. Cette séparation pose un problème majeur au chercheur qui veut rendre intelligibles ces archives. Quelques exemples des représentations relatives aux villas des Médicis et à leurs grottes peuvent nous aider à comprendre l'importance de ces documents, mais aussi la difficulté de l'interprétation de ces témoignages.

Cette documentation du fonds Regie possessioni concerne en effet tous les jardins des villas des Médicis : ainsi nous y trouvons des plans dédiés à Poggio a Caiano ${ }^{31}$, Topia (où sont mentionnées des statues et des grottes) ${ }^{32}$, Lapeggi $^{33}$, Cafaggiolo ${ }^{34}$, Poggio imperiale ${ }^{35}$, Careggi ${ }^{36}$, ce qui est particu-

di Toscana. Écarté de ce poste lors de la première occupation française en 1799, il le retrouva en 1801 auprès de Louis $I^{\text {er }}$ de Bourbon. Il en fut de nouveau privé par les Français en 1808 et le récupéra définitivement en 1814 jusqu’à sa mort en 1818 .

30. Boni, 1816.

31. ASFi, Scrittoio delle regie possessioni, t. 1-6.

32. ASFi, Scrittoio delle regie possessioni, t. 2-4.

33. ASFi, Piante delle regie possessioni, t. 3- 9 et t. 3-14.

34. ASFi, Scrittoio delle regie possessioni, t. 5-3 (daté par un document annexe de 1629).

35. ASFi, Piante delle regie possessioni, t. 9 et 9-1 ; Mignani Galli, I986. ASFi, Scrittoio regie possessioni, t. 258.

36. ASFi, Scrittoio delle regie possessioni, t. 8-1, t. 8-3, Piante topografiche, 68. Voir : Contorni, 1992 , p. $27-33$ 
lièrement intéressant car ils apportent des informations sur des lieux qui, de nos jours, ont été fortement urbanisés, notamment par la construction d'un hôpital moderne. On y trouve aussi les gravures de l'ancienne villa Médicis de Pratolino (détruite) et de son domaine, publiées par Bernardo Sansone Sgrilli dans sa Descrizione della regia villa, fontane, e fabbriche di Pratolino $(1742)^{37}$.

En outre, ce fonds conserve des représentations se rapportant à la villa de Castello $^{38}$ et de Petraia ${ }^{39}$ : une vue très intéressante représente les fontaines et la grotte de Castello (Fig. VIII, cahier couleur) ${ }^{40}$, une autre encore - dans laquelle nous reconnaissons la villa de Petraia, même si l'inscription la présente comme un plan de Castello ${ }^{41}$ - est ornée d'un Bacchus qui rappelle une iconographie assez répandue dans les jardins des villas florentines de l'époque. Le dieu est alors nu et potelé ; souvent à califourchon sur un tonneau qui sert de fontaine, il boit et offre du vin aux invités et, dans certains cas, il est au centre d'une véritable bacchanale ${ }^{42}$. Elle avait été associée au petit bronze de Jean Bologne (Musée national du Bargello, Florence, vers 1582), puis à des statues monumentales de jardin qui étaient situées au cœur de domaines viticoles, comme le Bacchus de Pierre de Francqueville (villa Bracci à Rovezzano, après 1579) ${ }^{43}$. Un choix iconographique qui s'apparente pleinement à un phénomène bien connu : le fait que l'unité fonctionnelle de la villa, du jardin et du domaine agricole - qui intervient notamment en Toscane - a un rôle déterminant

37. ASFi, Scrittoio delle regie possessioni, Piante topografiche 273 ; Piante delle regie possessioni, t. 1-19; Zangheri, 1987, vol. II Atlante, p. 156, fig. 311 ; Zangheri, 1996.

38. ASFi, Scrittoio delle regie possessioni, t. 2-2/2 à 2-2/4 ; t. 2-6.Voir aussi Acidini-Galletti, 1992, p. 81.

39. ASFi, Scrittoio delle regie possessioni, t. 2-7/1. Concernant les travaux réalisés à Petraia, après l'ère de la régence : Acidini-Galletti, 1992, p. 169- 186.

40. ASFi, Piante delle regie possessioni, tome 2, n 10, Florence, villa de Castello, XvIII ${ }^{\mathrm{e}}$ siècle, 485 $\mathrm{x} 760$, coll. T.

41. ASFi, Piante delle regie possessioni, t. 2-2/1. Bien que le cartouche se réfère à la « descrizione geografica di tutti i beni che nel presente stato gode e possiede il S.Gran Duca nostro Signore nella sua fattoria di Castello " [description géographique de tous les biens dont jouit et possède le GrandDuc notre Seigneur dans sa ferme Castello dans l'état actuel], le dessin fait référence à la villa et au jardin de la Petraia. C'est ainsi qu'il est répertorié (Veduta della villa di Petraia in un disegno del 1697) dans Chiostri, 1972, p. 36-tav. XIII, Acidini-Galletti, 1992, p. 170, Acidini-Galletti, 1995, p. 40. Encore une fois, le fait que la représentation graphique soit séparée de toute relation technique, rend difficile la juste compréhension de cette singularité.

42. Cicali, 2018.

43. Conigliello, 1999 ; Pegazzano, 1999 ; Cicali, 2017. 
dans la modélisation anthropique du territoire. Cette invention avait été tellement appréciée qu'elle avait été recommandée par le frère Agostino Del Riccio comme l'ornement d'un jardin idéal d'après les modèles florentins contemporains ${ }^{44}$, et elle avait aussi été proposée en France par les ingénieurs Francini, une fois qu'ils avaient émigré de Pratolino à la cour d'Henri $\mathrm{IV}^{45}$. Des Bacchus, antiques ou modernes, se trouvaient aussi à Florence, dans des grottes domestiche ${ }^{46}$, et dans les collections d'autres villas des Médicis $^{47}$, mais aucune explication n'est donnée par rapport à sa présence dans la représentation qui nous intéresse ici. Comme nous ne pouvons plus compter sur le document technique autrefois associé au dessin pour comprendre sa présence, nous devons souligner que, dans ces mêmes domaines, on produisait du vin dont la commercialisation avait été relancée, comme le montrent des plans contemporains des vignobles de Castello et des autres villas, conservés dans le même fonds (Fig. 2) ${ }^{48}$. La viticulture était particulièrement importante en Toscane, puisqu'à partir de la seconde moitié du XvII ${ }^{\mathrm{e}}$ siècle, en raison des bas prix des céréales, elle était une source de revenus essentielle. Dans les régions du Chianti, du Pomino, du Valdarno Superiore et de Carmignano, le vignoble toscan fut l'objet de la première dénomination d'origine contrôlée, souhaitée par le grand-duc Cosme III de Médicis en $1716^{49}$. La présence de Bacchus semble donc renouveler et poursuivre l'intention des œuvres littéraires telles que le Bacco in Toscana de Francesco Redi $(1685)^{50}$ : c'était un double hommage à la noble statuaire florentine qui avait orné ces parcs et à l'usage pratique qu'on voulait désormais leur donner.

\footnotetext{
44. "Attorno a dette grotte vi sieno botti in marmo, et sopra ogni botte vi stia su un giovinetto coronato come Bacco, et dette botti deon gettare sempre acqua " [Autour de ces grottes, qu'il y ait des barils de marbre, et au-dessus de chaque baril, il un jeune homme couronné comme Bacchus, et ces barils doivent toujours cracher de l'eau], Detlef Heikamp, p. 110 ; Del Riccio, Agricoltura sperimentale.

45. Sur les fontaines et fontainiers sous Henri IV voir Bresc-Bautier, 1990 ; pour Le Château-Neuf de Saint-Germain-en-Laye : Longo, 2002 ; Lurin, 2010 ; enfin au sujet de la gravure (Paris, INHA, gravure d'A. Bosse) : Lurin, 2017.

46. Rinaldi, 2001.

47. Capecchi, Lepore, Saladino, 1979, p. 22.

48. ASFi, Scrittoio delle regie possessioni, t. 2-5. Plusieurs plans des différents domaines indiquent les vins produits.

49. Ciuffoletti, 2016 ; Martelli, 2018.

50. Redi, 1685. L'ouvrage de l'académicien de la Crusca Francesco Redi est un éloge du vin toscan prononcé par Bacchus lui-même ; ce n'est pas un hasard s'il était souvent offert par les Médicis avec des vins qu'ils envoyaient comme cadeau diplomatique.
} 


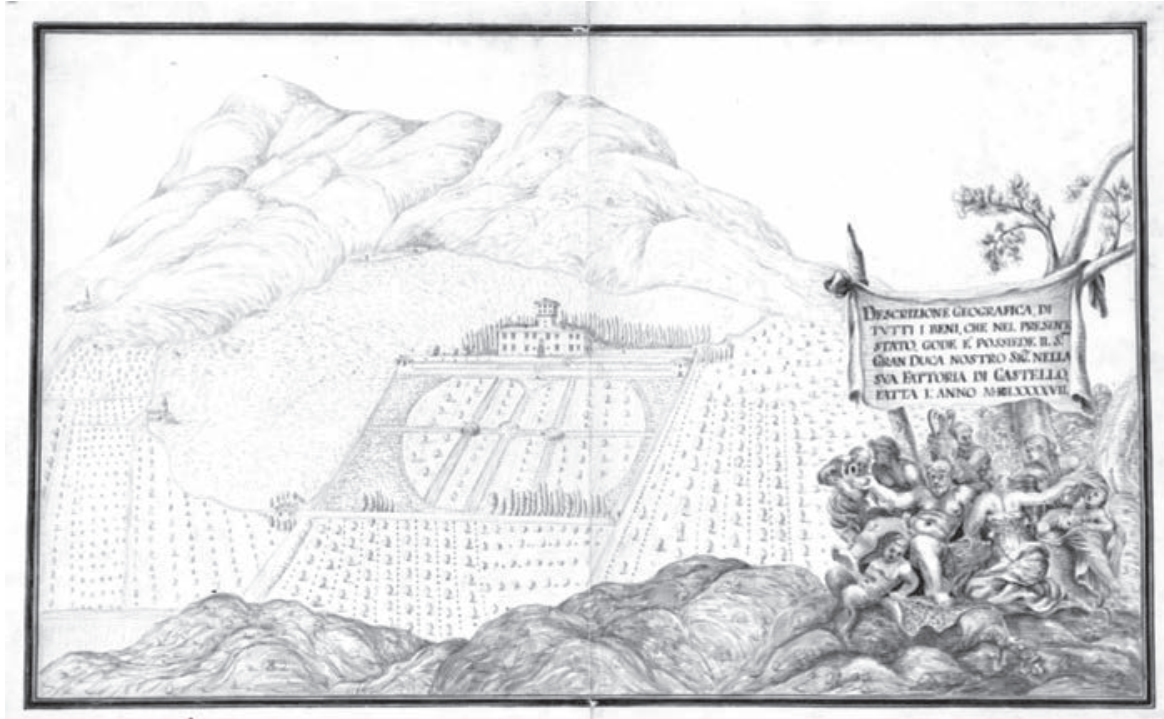

Fig. 2. - Castello : plan du domaine

Florence, Archives d'État, t. 2, n 2/1 (xvIII siècle).

\section{Conclusion}

L'analyse des archives conservées dans les deux fonds que nous avons signalés montre clairement l'importance d'une documentation très hétérogène par ses dimensions, ses techniques et ses fonctions, qu'il s'agisse des rapports techniques manuscrits ou des représentations graphiques. Ces documents ont été produits à partir de la dernière période des Médicis, à un moment où les grottes et les jardins étaient encore entretenus, jusqu'au temps des Habsbourg-Lorraine quand, pour des raisons économiques, les princes n'investissaient plus dans les grands domaines, s'interrogeaient sur leur utilité et commençaient à les démanteler. Grâce à ces sources tardives et indirectes, nous pouvons connaître l'évolution et la conservation des grottes, mais aussi comprendre quelles activités, ludiques ou commerciales, se déroulaient encore dans ces endroits au XVIII ${ }^{\mathrm{e}}$ siècle. 


\section{Sources}

\section{Florence, Archives d'État (ASFi)}

- Scrittoio delle Fortezze e Fabbriche, Fabbriche lorenesi :

- 1991, n 48 : Poggio Imperiale, villa, disegno di una tromba a acqua, 1779.

- 2010, n 114 : Poggio a Caiano, villa, pianta con la localizzazione di alcune macchine giocose, 1791.

- 2015, n⿳5 59 : Pratolino, villa, colonne di Verde antico della grotta, 1794.

- 2072, nº 20 : Poggio Imperiale, villa, fontana nel primo cortile (due disegni), 1820.

- 2058, n 3 : Castello, giardino e villa, 1816 (pianta, castello, giardino con grotta).

- 2077, no 9 1/4 : Firenze, Giardino di Boboli, collegamenti del condotto reale ad una abitazione privata adiacente la grotta, 1821 (abitazione adiacente alla grotta)

- 2081, n 32 : Castello, villa, pianta del giardino e annessi, 1822 (pianta, castello, giardino con grotta).

- Piante delle regie possessioni :

- tome II, $n^{\circ} 10$, Firenze-Castello, villa, XVIII ${ }^{\mathrm{e}}$ siècle, 485 x 760, coll. T.

- tome III, n 9, Apeggi, fattoria, (villa e giardino), XVIII ${ }^{\mathrm{e}}$ siècle, 625 x 865, coll T.

- tome IX, ${ }^{\circ} 1$, Firenze, Poggio imperiale, fattoria (villa), XVIII ${ }^{\mathrm{e}}$ siècle, $475 \mathrm{x}$ 665 , coll. F.

- tome IX, XVIII ${ }^{\mathrm{e}}$ siècle, Firenze, Poggio imperiale, Pianta generale di tutti $i$ poderi e fabbriche della fattoria di Poggio Imperiale.

- Scrittoio delle regie possessioni :

- tome I, n 6, Poggio a Caiano (jardin), Xviır siècle, 545 x 415.

- tome I, $\mathrm{n}^{\circ} 19$, Pratolino (villa et jardin), xvini ${ }^{\mathrm{e}}$ siècle. 333 x 465, coll. F.

- tome II, $n^{\circ} 2 / 2$, XVIII ${ }^{\mathrm{e}}$ siècle, 295 x 460, coll. T.

- tome II, $n^{\circ}$ 2/3, Castello, villa, xvini ${ }^{\mathrm{e}}$ siècle, 190 x 260, coll. T.

- tome II, n 2/4, Castello, XviII ${ }^{\mathrm{e}}$ siècle, 405 x 550, coll T. ;

- tome II, $\mathrm{n}^{\circ}$ 4, Topaia, (villa, casino del podere), XVIII ${ }^{\mathrm{e}}$ siècle, 485 x 760, coll.T.

- tome II, n 5, Castello, (villa, vigne), XviII siècle, 495, x 755.

- tome II, n 6, Castello, Petraia, Topaia (villas), XviII ${ }^{\mathrm{e}}$ siècle, 495 x 770, coll. $\mathrm{T}_{\text {. }}$

- tome II, $\mathrm{n}^{\circ}$ 7/1, Petraia, (villa et jardin), xviII ${ }^{\mathrm{e}}$ siècle, 485, x 765, coll. T.

- tome III, $\mathrm{n}^{\circ} 14$, Apeggi (villa et jardin), XVIII ${ }^{\mathrm{e}}$ siècle, 710 x 720, coll. T.

- tome V, no 3, Cafaggiolo (villa), Frosino Zampogni, 1629, 560 x 400.

- tome VIII, n 1, Careggi, (villa), Giovannozzo Giovannozzi, 1696, 485 x

375, coll. $\mathrm{T}$.

- tome VIII, n 3, Careggi, fattoria (villa), Giovannozzo Giovannozzi, 1696, $485 \times 375$, coll. T. 
Piante topografiche, $\mathrm{n}^{\circ} 273$ (Pratolino) ; n² 258 (Poggio imperiale villa); $\mathrm{n}^{\circ} 68$ (Careggi).

Paris, Institut national d'Histoire de l'Art (INHA) : eau-forte de Abraham Bosse (Tommaso Francini inventor), Modèle de fontaine avec un Bacchus, 1624.

De Caus Salomon, Les raisons des forces mouvantes, avec diverses machines tant utiles que plaisantes, Ausquelles sont adjoints plusieurs desseings de grottes et fontaines. Augmentées de plusieurs figures, avec le discours sur chacune, par Salomon de Caus, ingenieur et architecte du roi, ches Hierosme Drouart, Paris, 1624.

Del Riccio Agostino, Agricoltura sperimentale, Biblioteca nazionale Centrale di Firenze (B.N.C.F.), III, c. 77.r.

Del Riccio Agostino, Del giardino di un re, dans Il giardino storico italiano. Problemi di indagine. Fonti letterarie e storiche, Leo S. Olschki, Florence, 1981, p. 59-123.

De' Vieri Francesco, Discorsi di M. Francesco de’ Vieri, detto il Verino secondo, cittadino fiorentino, delle maravigliose opere di Pratolino, Marescotti, Florence, 1586.

Gualteoritti Raffaello, Vaghezze sopra Pratolino, Giunti, Florence, 1579.

Montaigne Michel de, Journal de voyage de Michel de Montaigne, édition présentée, établie et annotée par François Rigolot, Presses universitaires de France, Paris, 1992.

Redi Franceesco, Il Bacco in Toscana-Ditrambo di Francesco Redi accademico della Crusca con Annotazioni, Pier Piero Martini, Florence, 1685.

\section{Bibliographie}

Acidini Luchinat Cristina, Galletti Giorgio, Le ville e i giardini di Castello e Petraia a Firenze, Pacini Eitore, Pise, 1992.

Acidini Luchinat Cristina, Galletti Giorgio, La villa e il giardino della Petraia a Firenze, Edifir, Florence, 1995.

Bardazzi Silvestro, Castellani Eugenio, La villa medicea di Poggio a Caiano, Edizioni del Palazzo, Prato, 1981.

Becattini Massimo (dir.), Parco Mediceo di Pratolino-Villa Demidoff- Una storia per immagini, Edizioni Polistampa, Florence, 2005.

Benzoni Gino, "Medici, Francesco I de’ ", dans Dizionario biografico degli Italiani, vol. Xlıx, Istito della Enciclopedia italiana, Rome, 1997, p. 726-734.

Berti Luciano, Il Principe dello Studiolo: Francesco I dei Medici e la fine del Rinascimento fiorentino, Edam, Florence, 1967.

Bonfioli Maria, "Boni, Onofrio ", dans Dizionario biografico degli Italiani, vol. XII, Istito della Enciclopedia italiana, Rome, 1970, p. 84-85.

Boni Onofrio, Elogio dell'abate Luigi Lanzi, Niccolò Capurro, Pisa, 1816. 
Bresc-Bautier Geneviève, "Fontaines et fontainiers sous Henri IV ", dans, Les arts au temps d'Henri $I V$, actes du colloque de Fontainebleau, Pau, Association Henri IV, 1990, p. 93-120.

Brunon Hervé, "Pratolino, arts des jardins et imaginaire de la nature dans l'Italie de la seconde moitié du Xvi ${ }^{\mathrm{e}}$ siècle ", thèse de l'université Paris 1, 2001.

Brunon Hervé et Mosser Monique, "Quelques topiques dans l'imaginaire des grottes en Europe ", dans Zangheri Luigi (dir.), Ville e Giardini Medicei in Toscana e la loro influenza nell'arte dei giardini, Leo S. Olschki, Florence, 2017, p. 13-36.

Capecchi Gabriella, Saladino, Vincenzo, Lepore, Lucia (dir.), La villa del Poggio Imperiale, L'Erma di Bretschneider, Rome, 1979.

Capecchi Gabriele, Ipotesi su Castello: l'iconografia di Niccolo Tribolo e il giardino delle origini (1538-1550), Edizioni Polistampa, Florence, 2017.

Castellini, Marta, "Il Ninfeo di Egeria (II sec.) e la Grotta degli Animali a Castello. Il ruolo del modello antico attraverso l'analisi dei disegni del GDSU », dans Ferretti Emanuela, Frommel Sabine, Giannozzi Alessandra et al. (dir.), "Il rinascimento delle grotte. Natura, arte, e architettura fra Italia e Francia nel Cinquecento ", Opus Incertum, n 4, 2018, p. 36-43.

Cazzato Vincenzo, Fagiolo, Marcello, Giusti, Maria Adriana (dir.), Atlante delle grotte e dei nifei in Italia- Toscana, Lazio, Italia meridionale e isole, Electa, Milan, 2001.

Chauvineau Hélène, "La cour des Médicis (1543-1737) », dans Boutier Jean, Landi Sandro, Rouchon Olivier (dir.), Florence et la Toscane, XIV-XIX siècles. Les dynamiques d'un État italien, Presses Universitaires de Rennes, Rennes, 2004, p. 287-302.

Chiostri Ferdinando, La Petraja: villa e giardino settecento anni di storia, Leo S. Olschki, Florence, 1972.

Cicali Giulia, "Le sculpteur Francesco Bordoni, collaborateur des Francini », dans Lurin Emmanuel et Rostaing Aurélia (dir.), " Hydraulique et fontaines ornementales en France (vers 1590-1640). Autour de Tommaso et Alessandro Francini ", Bulletin monumental, t. 175-4, 2017, p. 357-367.

Cicali Giulia, "Su Bacco, sul vino, sul dono (nella Toscana granducale) ", dans Gennai Paolo (dir.), Carmignano, il vino del Granduca, Editori dell'Acero, Empoli, 2018, p. 67-78.

Ciuffoletti Zefiro, "Cosimo III e la denominazione dei vini di qualità nel contesto europeo ", dans Ciuffoletti Zefiro (dir.), Terre, uve, vini. La denominazione dei vini di qualità nella Toscana medicea e il contesto europep, Polistampa, Florence, 2016, p. 13-42. 
Conforti Claudia, "Pratolino come mito della conoscienza e alfabeto figurato dell'immaginario ", dans Fagiolo Marcello (dir.), La città effimera e l'universo artificiale del giardino, éd. Officina, Roma, 1980, p. 183-192.

Conigliello Lucilla, "Pietro Francavilla e il giardino segreto di Villa Bracci: una nota del 1750 », Paragone, 1999, n 27 (595), p. 95-102.

Contorni Gabriella, La villa medicea di Careggi, Becocci/Scala, Florence, 1992.

Dezzi Bardeschi Marco, "Da Pratolino che minaccia rovina... ", dans Dezzi Bardeschi Marco, Vezzosi Alessandro et Zangheri Luigi (dir.), Il giardino d'Europa. Pratolino come modello della cultura Europea, Mazzotta, Milan, 1986, p. 107-118.

Di Croce Alessandra, "Onofrio Boni architetto intendente: gli scritti teorici ", Neoclassico, $\mathrm{n}^{\circ} 21,2002$, p. 27-48.

Di Croce Alessandra, «Onofrio Boni e lo Scrittoio delle Regie Fabbriche: il mestiere dell'architetto a Firenze tra Sette e Ottocento ", Ricerche di storia dell'arte, $\mathrm{n}^{\circ} 84,2004$ (2005), p. 25-34.

Fasano Guarini Elena, "Cosimo I de' Medici granduca di Toscana ", dans Dizionario Biografico degli Italiani, vol. xxx, Istito della Enciclopedia italiana, Rome, 1984, p. 30-48.

Ferretti Emanuela, Frommel Sabine, Giannozzi Alessandra et al (dir.), «Il rinascimento delle grotte- Natura, arte, e architettura fra Italia e Francia nel Cinquecento ", Opus Incertum, no 4, 2018.

Ferretti Emanuela, Frommel Sabine, Giannozzi Alessandra, Mozzo Marco, (dir.), " Il rinascimento delle grotte. Natura, arte, e architettura fra Italia e Francia nel Cinquecento ", Opus Incertum, n 4, 2018.

Galetto Guido, La villa medicea di Poggio a Caiano : tra l'Atene degli Acciaiuoli ed il Granducato della Baciocchi, Gangemi editore SpA international, Roma, 2018.

Longo Marina, "Tommaso Francini, ingegnere, scenografo, "honorable homme" fiorentino alla corte di Francia (1598-1651)", Teatro e storia, 2002-2003, n²4, p. $377-425$.

Lurin Emmanuel (dir.), Le Château-Neuf de Saint-Germain-en-Laye, Les Presses franciliennes, Saint-Germain-en-Laye, 2010.

LuRIN Emmanuel, " Tommaso Francini inventor. Les modèles de grottes et de fontaines (1622-1624) et l'art du fontainier florentin en France », dans LuRIN Emmanuel et Rostaing Aurélia (dir.), "Hydraulique et fontaines ornementales en France (vers 1590-1640). Autour de Tommaso et Alessandro Francini ", Bulletin monumental, t. 175-4, 2017, p. 325-356.

Martelli Francesco, "Dai "Provveditori" allo "Scrittoio". Note sulle origini dello scrittoio delle fortezze e fabbriche nel Cinquecento ", Opus Incertum, $n^{\circ} 1,2006$, p. 75-83. 
Martelli Francesco, «La politica vinicola di Cosimo III dei Medici e i bandi del 1716 », dans Gennai Paolo (dir.), Carmignano, il vino del Granduca, Editori dell'Acero, Empoli, 2018, p. 51-66.

Medri Litta, Mazzoni Paolo, De Vico Fallani, Massimo, La villa di Poggio a Caiano, Becocci, Florence, 1986.

Mignani Galli Daniela, "Un'idea di giardino moderno per un giardino prospettico ", dans Vezzosi Alessandro (dir.), Il giardino romantico, Alinea, Florence, 1986, p. 46-55.

Morel Philippe, Les Grottes maniéristes en Italie au XVT siècle : théâtre et alchimie de la nature, Macula, Paris, 1998.

Pegazzano Donatella, «Il giardino Bracci a Rovezzano : precisazioni e aggiunte alla scultura di Pietro Francavilla ", Paragone, 1999, n 27 (595), p. 63-94.

Rinaldi Alessandro, "Grotte domestiche a Firenze ", dans Cazzato Vincenzo, Fagrolo Marcello, Giusti Maria Adriana (dir.), Atlante delle grotte e dei ninfei in Italia, Toscana, Lazio, Italia meridionale e isole, Electa, Milan, 2001, p. 22-33

Rombai Leonardo, Toccafondi Diana, Vivoli Carlo, I fondi cartografici dell'Archivio di Stato di Firenze: I Miscellanea di Piante, Leo S. Olschki, Florence, 1987.

VAldaré Giovanni, Bibliografia storico-ragionata della villa medicea e della sua gente, Alinea, Florence, 2003.

ZANGHERI Luigi, «I giardini d'Europa : una mappa della fortuna medicea nel XVI e XViI secolo ", dans Dezzi Bardeschi Marco, Vezzosi Alessandro, Zangheri Luigi (dir.), Il giardino d'Europa. Pratolino come modello della cultura Europea, Mazzotta, Milan 1986, p. 82-91.

Zangheri Luigi, "Lartificio del paesasggistico nel Parco di Joseph Frietsch ", dans Dezzi Bardeschi Marco, Vezzosi Alessandro, Zangheri Luigi (dir.), Il giardino d'Europa. Pratolino come modello della cultura Europea, Mazzotta, Milan, 1986, p. 119-123.

Zangheri Luigi, "Salomon de Caus e la fortuna di Pratolino nell'Europa del primo Seicento ", dans Vezzosi Alessandro (dir.), La fonte delle fonti. Iconografia degli artifizi d'acqua, Florence Alinea, 1985, p. 35-43.

Zangheri Luigi, "Sette planimetrie inedite per Pratolino ", Antichità viva, n 34, 1995-4 (1996), p. 22-25.

Zangheri Luigi, Le ville medicee in Toscana nella lista del Patrimonio Mondiale, Olschki, Florence, 2015.

Zangheri Luigi, Pratolino: il giardino delle meraviglie, Gonnelli, Florence, (1979) 1987. 


\section{L'autrice}

Docteure en Histoire de l'art, Giulia Cicali a soutenu en 2012 une thèse consacrée au sculpteur Francesco Bordoni, sous la direction des professeurs Alessandro Angelini et Pascal Julien (Università degli Studi di Siena, Université de Toulouse II-Le Mirail). Elle a enseigné en Italie et en France et étudie actuellement les sculpteurs florentins qui ont travaillé à Paris, leurs réseaux, le mécénat des Italiens installés en France, la politique des arts en Toscane et à la cour française aux $x v^{e}$ et $x v I^{e}$ siècles. Elle prépare une thèse de post-doctorat, sous la direction du professeur Sabine Frommel (EPHE), intitulée « Entre duché de Toscane et royaume de France : la statuaire dans l'espace urbain et les jardins palatiaux ».Contact : cicali.giulia@gmail.com 